\title{
Hyperbolic axial dispersion model for heat exchangers
}

\author{
Ranjit Kumar Sahoo a, Wilfried Roetzel ${ }^{\text {b,* }}$ \\ ${ }^{a}$ Mechanical Engineering Department, Regional Engineering College, Rourkela 769008 , India \\ b Institute of Thermodynamics, University of the Federal Armed Forces Hamburg, D-22039 Hamburg, Germany
}

\begin{abstract}
Flow maldistribution in heat exchangers for steady-state and transient processes can be described by dispersion models. The traditional parabolic model and the proposed hyperbolic model which includes the parabolic model as a special case can be used for dispersive flux formulation. Instead of using the heuristic approach of parabolic or hyperbolic formulation, these models can be quantitatively derived from the axial temperature profiles of heat exchangers. In this paper both the models are derived for a shell-and-tube heat exchanger with pure maldistribution (without back mixing) in tube side flow and the plug flow on the shell side. The Mach number and the boundary condition which plays a key role in the hyperbolic dispersion have been derived and compared with previous investigation. It is observed that the hyperbolic model is the best suited one as it compares well with the actual calculations. This establishes the hyperbolic model and its boundary conditions.
\end{abstract}

Keywords: Dispersion; Heat exchangers; Modeling

\section{Introduction}

Theoretical analysis of heat exchangers has received considerable attention during the last decade. On one side the steady-state analyses have stressed the need for theoretical and experimental evaluation of heat exchanger performance during normal operation. On the other hand the transient studies have brought out the response feature due to off-normal behaviour which are of immense importance for impacting proper control strategy. Even though the advent of high speed computing system has made the complete numerical simulation of heat exchangers possible, still the need for understanding the mechanism of transport phenomenon remains equally important. Understanding of transport phenomenon will reveal the experimental procedure to be applicable for design and control of heat exchangers. A significant breakthrough in this direction has been achieved using the concept of apparent axial dispersion phenomenon in mass transfer by Taylor [1,2] and Danckwerts [3]. From heat and mass transfer analogy it is established $[4,5]$ that the same concept can be used for heat transfer as well. The main attraction of axial dispersion model is its power to effectively amend the prediction of plug flow model while still retaining the latter's simplicity of unidirectional characteristics. Due to this simplicity, the axial dispersion model has generated a greater interest of applicability in a wide variety of dispersion problems in association with chemical reactor, heat exchangers and various separation processes [6].

Most convective heat and mass dispersion phenomena strongly resemble molecular diffusion, only that they have absolutely different times, velocities and space scales. The relationship of these scales to other process scales may be completely different than for molecular diffusion. Therefore the application of Fourier's law to describe convective dispersion phenomenon often is not justified and leads to erroneous results.

In their pioneering works, Taylor [1,2] and Danckwerts [3] introduced axial dispersion model based on Fourier's law to describe flow maldistribution or back mixing in flow systems. This leads to the boundary conditions of Danckwerts [3] for parabolic dispersion model. Fourier's law implies an infinite propagation 


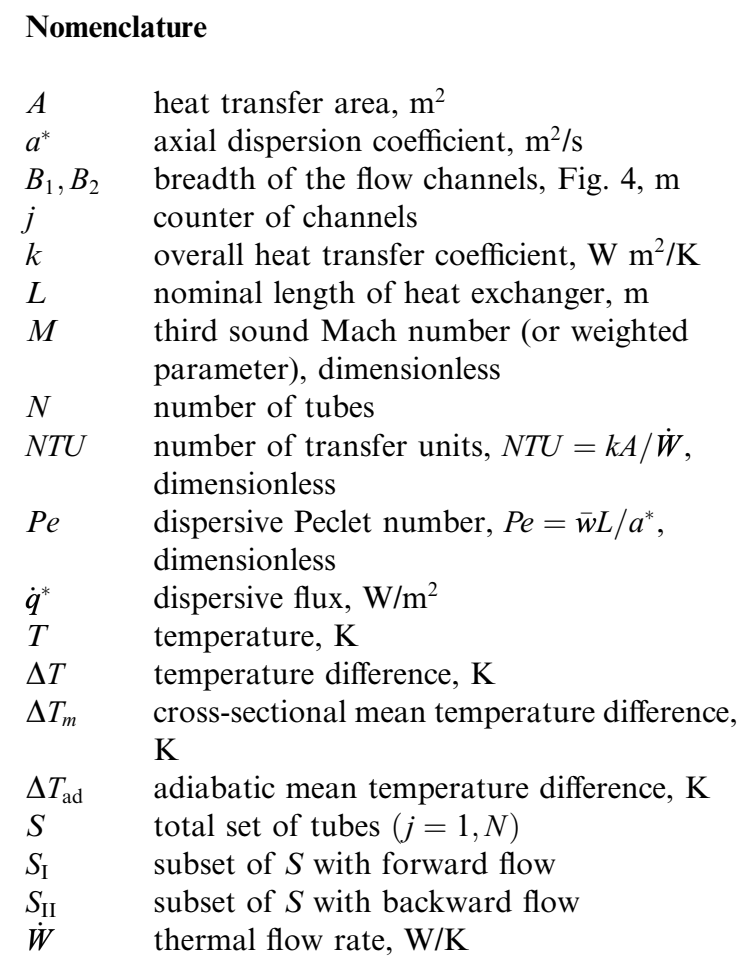

$\begin{array}{ll}w & \text { flow velocity, } \mathrm{m} / \mathrm{s} \\ \bar{w} & \text { mean flow velocity, } \mathrm{m} / \mathrm{s} \\ x & \text { space coordinate, } \mathrm{m}\end{array}$

Greek symbols

$\lambda^{*} \quad$ dispersive conductivity, W/m K

$\theta \quad$ dimensionless temperature, $\theta=\left(T-T_{2}^{\prime}\right) /$ $\left(T_{1}^{\prime}-T_{2}^{\prime}\right)$

$\varphi \quad$ dimensionless dispersive flux, $\varphi=\dot{q}^{*} /$ $\left[\lambda^{*}\left(T_{1}^{\prime}-T_{2}^{\prime}\right) / L\right]$

$\xi \quad$ dimensionless spatial variable, $\xi=x / L$

\section{Subscripts}

1 fluid 1

2 fluid 2

back backward flow in tubes

$f \quad$ adiabatic mean

forward forward flow in tubes

hyp hyperbolic

$m$ cross-sectional mean

par parabolic

Superscripts

,$\quad$ inlet

$+\quad$ at the boundary with higher value of $\xi$

- $\quad$ at the boundary with lower value of $\xi$ velocity of disturbances, which is a good approximation for normal heat conduction. However, maldistribution effects will propagate with a finite velocity which has the order of mean flow velocity. The parabolic model for the description of axial dispersion is questionable, since time and velocity scales of the axial dispersion process are essentially different from those for ordinary molecular diffusion; they are comparable to the characteristic time such as residence time. To take finite propagation velocities into account the law of Fourier has to be replaced by the heat conduction law of Chester [7]. This leads to the hyperbolic heat conduction equation, known as hyperbolic dispersion model. This new dispersion model is more general and can be reduced to parabolic model as a special case. Additionally, hyperbolic model has the ability to distinguish between real axial mixing, diffusion or conduction on the one hand and other types of maldistribution on the other hand.

An extension of the axial dispersion model was first proposed by Roetzel and Spang [8,9] by incorporating Chester's constitutive equations for fluid flow. In their model, a parameter of finite propagation velocity appears which they named as the "third sound wave" where the "second sound wave" is the molecular conduction wave $[10,11]$. Shortly after Roetzel and Spang, and independent of them, Westerterp et al. [12,13] proposed and analysed a similar model for longitudinal mass dispersion in chemical reactors following Taylor's approach [1]. As compared with Chester's equation, the latter model for constitutive mass dispersion equation additionally contains the derivation of source term with respect to concentration driving potential for chemical reaction and a parameter for velocity asymmetry. This wave model was subsequently applied to tubular reactors [14,15]. The hyperbolic dispersion model [8,9] based on Chester's equation has been successfully applied to heat exchangers [16]. Further, Roetzel et al. [17] validated the hyperbolic heat flux equations with different examples and also proposed boundary conditions for subsonic, sonic and supersonic flow regimes by defining the Mach number as the ratio of fluid velocity to thermal wave velocity. However, these boundary conditions have been modified and generalised by Sahoo and Roetzel [18] based on an elegant analytical approach. In a recent work by Roetzel and Na Ranong [19] it is shown for the first time that in a shell-and-tube heat exchanger with pure tube side maldistribution (without axial mixing) the longitudinal mean temperature profiles can accurately be described with the hyperbolic dispersion model. The derivations given in [19] are further refined in the present work.

Most of the experimental and theoretical investigations of hyperbolic axial dispersion in heat exchangers that have been published are based on the heuristic approach of using Chester's equation and verifying the 


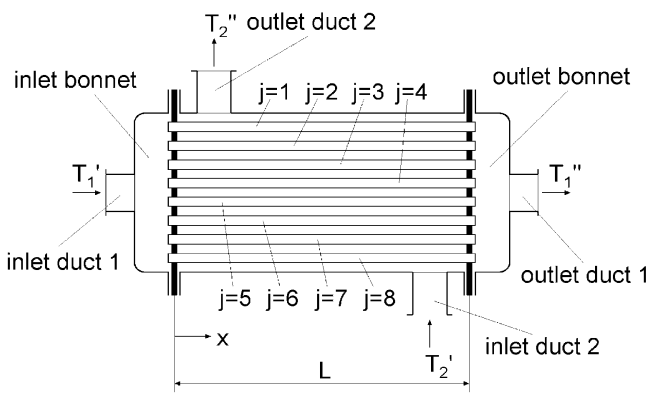

Fig. 1. Schematic of shell-and-tube heat exchanger with tube channels $(j=1, \ldots, 8)$.

result by numerical calculation. Earlier investigators have not paid much attention to uncovering the underlying phenomena through a detailed mathematical treatment which will lead to hyperbolic dispersion model and its boundary conditions from the constitutive equations of heat exchanger.

In the present analysis, as in [19], a simple shell-andtube heat exchanger is considered where maldistribution is present only in the tube side flow as shown in Fig. 1. From the behaviour of the mean temperature profiles, the parabolic and hyperbolic dispersion models are derived by using Taylor's expansion. The parameters and boundary conditions are derived from the constitutive equations of the heat exchanger. It has been observed that these boundary conditions are equal to the boundary conditions [18] that have been derived entirely from a different approach. It is further observed that the mean temperature profile based on the hyperbolic model accurately matches with the actual calculation of heat exchanger. Since the derivations in this paper are more general, it will lead to a better understanding of the mechanisms responsible for the characteristics of the axial dispersion phenomena in heat exchangers.

\section{Governing equations in shell-and-tube heat exchanger with maldistribution in tube side}

In the shell-and-tube heat exchanger, a constant velocity of tube side fluid is usually assumed in all the tubes. Actually, the velocity may vary from tube to tube due to disadvantageous geometry of the inlet duct, outlet duct and bonnets. On the shell side, plug flow is assumed. For simplicity the overall heat transfer coefficient is assumed to be uniform. This type of steadystate maldistribution due to flow non-uniformity in tube side gives dispersion which is investigated in this paper.

Under the realistic assumption of plug flow inside each of the $N$ tubes, the tube side equation is,

$\frac{\mathrm{d} \theta_{1 j}}{\mathrm{~d} \xi}+\frac{k A / N}{\left(\dot{W}_{1} / N\right)\left(w_{1 j} / \bar{w}_{1}\right)}\left(\theta_{1 j}-\theta_{2}\right)=0$.
This equation may be rewritten as

$\frac{w_{1 j}}{\bar{w}_{1}} \frac{\mathrm{d} \theta_{1 j}}{\mathrm{~d} \xi}+N T U_{1}\left(\theta_{1 j}-\theta_{2}\right)=0$.

The second term of Eq. (2) represents the local heat flux at the $j$ th tube due to shell side fluid. Summing up all $N$ equations and dividing by $N$ gives

$\frac{1}{N} \sum_{j=1}^{N} \frac{\mathrm{d}}{\mathrm{d} \xi}\left(\frac{w_{1 j}}{\bar{w}_{1}} \theta_{1 j}\right)+N T U_{1}\left(\frac{1}{N} \sum_{j=1}^{N} \theta_{1 j}-\theta_{2}\right)=0$,

where the second term represents the average local heat flux associated with all the tube side flow and this must be equal to the lateral flux associated with the shell side fluid. Thus the shell side energy equation is given as

$\frac{\mathrm{d} \theta_{2}}{\mathrm{~d} \xi}+N T U_{2}\left(\frac{1}{N} \sum_{j=1}^{N} \theta_{1 j}-\theta_{2}\right)=0$.

For counter flow $N T U_{2}>0$, for cocurrent flow $N T U_{2}<0$ and for condensation or evaporation $N T U_{2}=0$.

The mean flow velocity inside the tube bundle in the direction of $\xi$ is always positive and can be expressed as $\bar{w}_{1}=\frac{1}{N} \sum_{j=1}^{N} w_{1 j}>0$.

For partial back flow which is also under consideration, flow velocities in some of the tubes are negative but the mean flow velocity is always positive.

Eqs. (2) and (4) generate $N+1$ equations which are to be solved by specifying the boundary conditions. For no back flow, the boundary conditions are given by Eqs. (6)-(8):

(i) For counter flow and cocurrent flow without back flow $\left(w_{1 j}>0 ; j=1, N\right)$

$\xi=0: \quad \theta_{1 j}=\theta_{1}^{-}=1$.

(ii) For counter flow $\left(\mathrm{NTU}_{2}>0\right)$

$$
\xi=1: \quad \theta_{2}=0 .
$$

(iii) For cocurrent flow $\left(N T U_{2}<0\right)$

$$
\xi=0: \quad \theta_{2}=0 .
$$

In case of back flow in some of the tubes $\left(\bar{w}_{1}>0\right)$, let the set of tubes $S_{\mathrm{I}}$ have the forward flow and the set $S_{\text {II }}$ have the backward flow. Thus, $S_{\mathrm{I}} \subset(j=1, N)$ and $S_{\text {II }} \subset(j=1, N)$; such that, $S_{\mathrm{I}} \cup S_{\text {II }}=S=(j=1, N)$ and $S_{\mathrm{I}} \cap S_{\text {II }}=0$.

The boundary conditions due to this partial back flow are given by Eqs. (9)-(12) by taking into account that the inlet and outlet bonnets are adiabatic mixing chambers:

(i) At the inlet cross-section

$$
\begin{aligned}
& \xi=0: \quad \sum_{j=1}^{N} \frac{w_{1 j}}{\bar{w}_{1}}\left(\theta_{1}^{-}-\theta_{1 j}\right)=0, \quad \theta_{1}^{-}=1, \\
& \text { and for } w_{1 j}>0 \Rightarrow \theta_{1 j}=\theta_{1, \text { forward }}=\text { const }\left(j \in S_{\mathrm{I}}\right) .
\end{aligned}
$$


(ii) At the outlet cross-section

$$
\xi=1: \quad \sum_{j=1}^{N} \frac{w_{1 j}}{\bar{w}_{1}}\left(\theta_{1 j}-\theta_{1}^{+}\right)=0,
$$

and for $w_{1 j}<0 \Rightarrow \theta_{1 j}=\theta_{1, \text { back }}=\theta_{1}^{+}=$const $\left(j \in S_{\text {II }}\right)$.

(iii) For counter flow $\left(N T U_{2}>0 ; \bar{w}_{1}>0\right)$

$$
\xi=1: \quad \theta_{2}=0 .
$$

(iv) For cocurrent flow $\left(N T U_{2}<0 ; \bar{w}_{1}>0\right)$

$$
\xi=0: \quad \theta_{2}=0 .
$$

In the heat transfer terminology there appear two mean temperatures: cross-sectional mean and adiabatic mean. The cross-sectional mean temperature is the arithmetic mean value of all tube side fluid temperatures at a location $\xi$. The adiabatic mean temperature is the weighted mean of tube side temperatures at a location $\xi$. The two mean temperatures are expressed as

$\theta_{1 m}=\frac{1}{N} \sum_{j=1}^{N} \theta_{1 j}$ and

$\theta_{1 f}=\frac{1}{N} \sum_{j=1}^{N} \frac{w_{1 j}}{\bar{w}_{1}} \theta_{1 j}$

The cross-sectional mean temperature $\theta_{1 m}$ is the driving potential for heat transfer whereas the adiabatic mean temperature is the actual exit fluid temperature. These two temperatures are related as

$$
\begin{aligned}
\theta_{1 f} & =\theta_{1 m}+\left(\theta_{1 f}-\theta_{1 m}\right) \\
& =\theta_{1 m}+\frac{1}{N} \sum_{j=1}^{N}\left(\frac{w_{1 j}}{\bar{w}_{1}}-1\right) \theta_{1 j} .
\end{aligned}
$$

The system of $N+1$ differential equations, Eqs. (2) and (4), can be solved numerically or analytically along with boundary conditions, Eqs. (6)-(12), to give the crosssectional and adiabatic mean temperature profiles. Using a suitable finite difference method the results obtained by Roetzel and $\mathrm{Na}$ Ranong [19] are shown in Figs. 2 and 3.
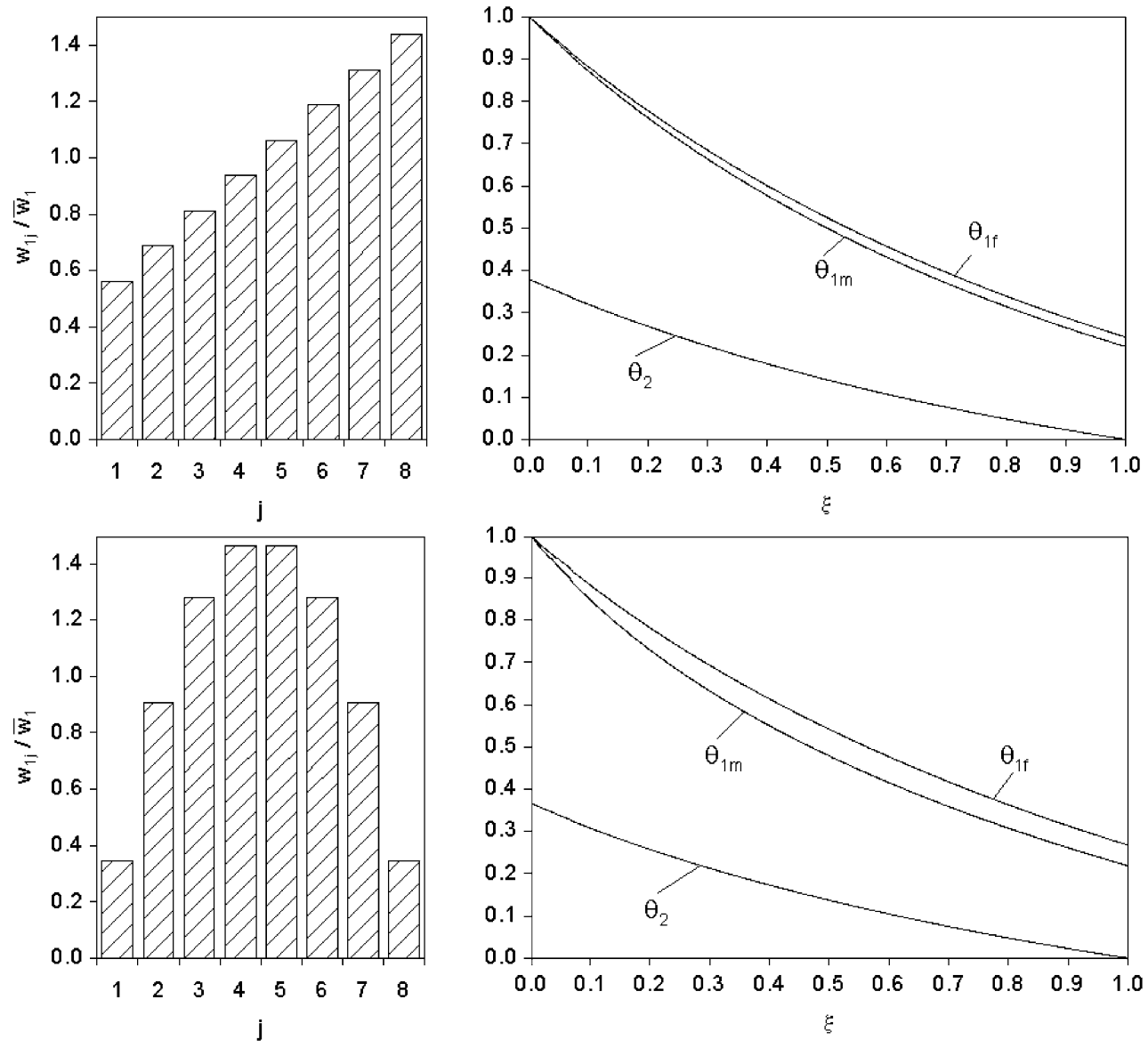

Fig. 2. Numerically calculated axial temperature profiles for maldistribution without back flow for $N T U_{1}=2$ and $N T U_{2}=1$. Top: linear velocity distribution; bottom: quadratic velocity distribution. 

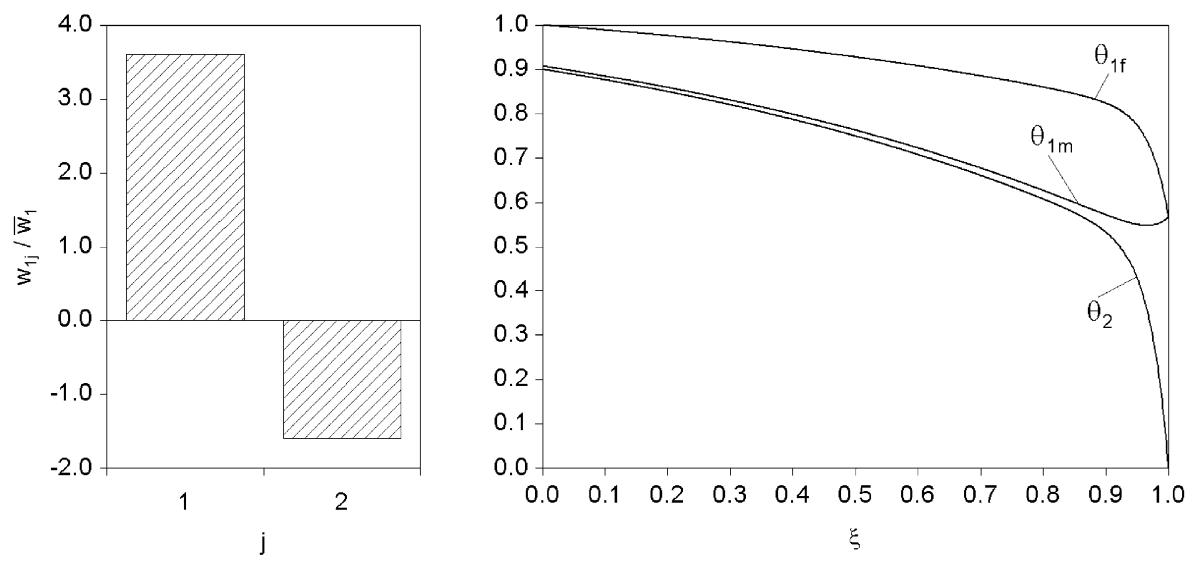

Fig. 3. Numerically calculated axial temperature profiles for two channels with back flow in one channel, $N T U_{1}=14.4$ and $N T U_{2}=30$.

At the inlet bonnet to the heat exchanger for no back flow, Eq. (6) indicates that both the mean values of inlet temperature to the tubes assume the value of $\theta_{1}^{-}$. Thus Eq. (6) and also Fig. 2 lead to

$\xi=0: \quad \theta_{1 f}=\theta_{1 m}=\theta_{1}^{-}=1$

At the outlet bonnet, as shown in Fig. 2, all fluid streams are mixed to leave at a common exit temperature $\theta_{1 f}$ which is different from $\theta_{1 m}$. Thus

$\xi=1: \quad \theta_{1 f} \neq \theta_{1 m}$.

The recirculation of fluid causes mixing of forward and back flows at the bonnets. At the inlet bonnet, Eq. (9) and also Fig. 3 indicate that the adiabatic mean temperature is equal to the inlet fluid temperature and the cross-sectional mean temperature drops suddenly. Thus

$\xi=0: \quad \theta_{1 f}=\theta_{1}^{-}=1 \neq \theta_{1 m}$.

At the outlet bonnet, Eq. (10) and also Fig. 3 show that both the mean temperatures are equal to the exit fluid temperature. Thus

$\xi=1: \quad \theta_{1 f}=\theta_{1 m}=\theta_{1}^{+}$.

These boundary conditions in terms of mean temperatures are used later to establish the boundary conditions for a hyperbolic model.

\section{Axial dispersion models}

The existence of dispersion terms can be derived by rearranging the governing equations for the heat exchanger in terms of the cross-sectional mean temperature which is the decisive temperature for heat transfer. Eqs. (3) and (4) can be rewritten in terms of their mean temperatures as:

$$
\begin{aligned}
& \frac{\mathrm{d} \theta_{1 f}}{\mathrm{~d} \xi}+N T U_{1}\left(\theta_{1 m}-\theta_{2}\right)=0 \\
& \frac{\mathrm{d} \theta_{2}}{\mathrm{~d} \xi}+N T U_{2}\left(\theta_{1 m}-\theta_{2}\right)=0 .
\end{aligned}
$$

Using Eq. (15), Eq. (20) can be expressed as

$\frac{\mathrm{d} \theta_{1 m}}{\mathrm{~d} \xi}+\frac{1}{P e} \frac{\mathrm{d} \varphi}{\mathrm{d} \xi}+N T U_{1}\left(\theta_{1 m}-\theta_{2}\right)=0$.

The local dimensionless dispersive flux at any location $(\xi)$ may be defined as

$$
\begin{aligned}
\varphi(\xi) & =\operatorname{Pe}\left[\theta_{1 f}(\xi)-\theta_{1 m}(\xi)\right] \\
& =\frac{P e}{N} \sum_{j=1}^{N}\left(\frac{w_{1 j}}{\bar{w}_{1}}-1\right) \theta_{1 j}(\xi) .
\end{aligned}
$$

Eq. (22) reveals that the maldistributed tube side flow can be treated as a plug flow stream with mean velocity $\bar{w}_{1}$ and temperature $\theta_{1 m}$. However, a correction term, known as dispersion, is necessary to account for the difference between two mean temperatures. The dispersion term may be recognised as a dispersive flux as shown in Eq. (22) to account for the maldistribution effect in tube side flow. Since Eq. (22) has to be expressed in terms of $\theta_{1 m}$ to take care of heat transfer, the dispersive flux should also be expressed as a function of the same variable.

The variables $\theta_{1 f}$ and $\theta_{1 m}$ are related to each other by a certain functional relationship. At the present context, it is not of interest to derive this relation. Here, a simple method [17] has been adopted to illustrate the difference between these two mean temperatures. Let the two streams of fluid with velocities $w_{1}$ and $w_{2}$ flow through two channels of widths $B_{1}$ and $B_{2}\left(B_{1}>B_{2}\right)$, respectively, as shown in Fig. 4. The temperature change for similar thermal boundary conditions for the channels (e.g., constant wall heat flux) can be found to be

$\Delta T_{1}>\Delta T_{2}$ for $w_{1}<w_{2}$. 


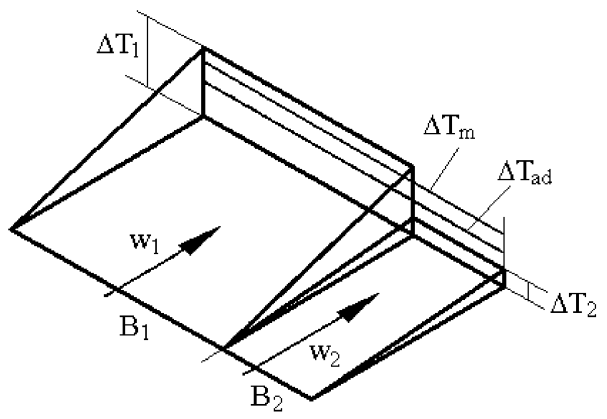

Fig. 4. Fluid flowing through adjacent channels with different temperatures and velocities: illustration of the difference between cross-sectional and adiabatic mean temperatures.

The cross-sectional mean temperature at the exit can be calculated as

$\Delta T_{m}\left(B_{1}+B_{2}\right)=\Delta T_{1} B_{1}+\Delta T_{2} B_{2}$.

The adiabatic mean temperature must take care of the weighted mean to yield

$\Delta T_{\mathrm{ad}}\left(B_{1} w_{1}+B_{2} w_{2}\right)=\Delta T_{1} B_{1} w_{1}+\Delta T_{2} B_{2} w_{2}$.

Hence the difference between the two mean temperatures can be derived as

$$
\begin{aligned}
& \Delta T_{m}-\Delta T_{\mathrm{ad}} \\
& \quad=\left(\Delta T_{1}-\Delta T_{2}\right)\left(\frac{B_{1}}{B_{1}+B_{2}}-\frac{B_{1}}{B_{1}+B_{2} w_{2} / w_{1}}\right) .
\end{aligned}
$$

Since both the terms on the right-hand side of this equation have the same sign, it can be inferred that the cross-sectional mean temperature changes faster than the adiabatic mean temperature and the case is changed for fluid losing heat. The sketches of the temperature profiles are shown in Fig. 5(i) and (ii). In the absence of lateral heat conduction through the wall, the nature of the temperature as shown in Fig. 5(ii) is consistent with Figs. 2 and 3.

Let $a c$ and $b c$ be vertical and horizontal lines, respectively, where the points $a, b$ and $c$ are situated on the mean temperature profile as shown in Fig. 5. Along the horizontal line the temperature $\theta_{1 f}$ at the location $\xi$ is equal to $\theta_{1 m}$ at the location $(\xi-1 / P e)$ where the horizontal distance is assumed to be $1 / P e$. Hence using Taylor's expansion, Eq. (23) can be written as

$$
\begin{aligned}
\varphi(\xi) & =P e\left[\theta_{1 m}(\xi-1 / P e)-\theta_{1 m}(\xi)\right] \\
& =-\frac{\mathrm{d} \theta_{1 m}}{\mathrm{~d} \xi}+\frac{1}{2 !} \frac{1}{P e} \frac{\mathrm{d}^{2} \theta_{1 m}}{\mathrm{~d} \xi^{2}}-\frac{1}{3 !} \frac{1}{P e^{2}} \frac{\mathrm{d}^{3} \theta_{1 m}}{\mathrm{~d} \xi^{3}}+\cdots
\end{aligned}
$$

A parabolic model can be obtained from Eq. (27) by neglecting the second- and higher-order terms to give

$\varphi=-\frac{\mathrm{d} \theta_{1 m}}{\mathrm{~d} \xi}$

The associated Peclet number $P e_{\text {par }}$ for this model can be estimated from the global energy balance in the heat exchanger.

The parabolic model given by Eq. (28) indicates that flux is equal to temperature gradient at the same location if the higher terms are neglected. If the higher order terms are appreciable a general proposition may be that the location of flux and temperature gradient may differ. This means that the very deletion of second and higher terms should be compensated by changing the location of flux. Thus

$\varphi\left(\xi+M^{2} / P e\right)=-\frac{\mathrm{d} \theta_{1 m}}{\mathrm{~d} \xi}$.

Here $M^{2}$ is a certain weighted parameter to decide the location of flux and this parameter has to be determined from the heat exchanger equations. This is the hyperbolic formulation which approaches the parabolic model in the limiting case of $M=0$. The hyperbolic model of

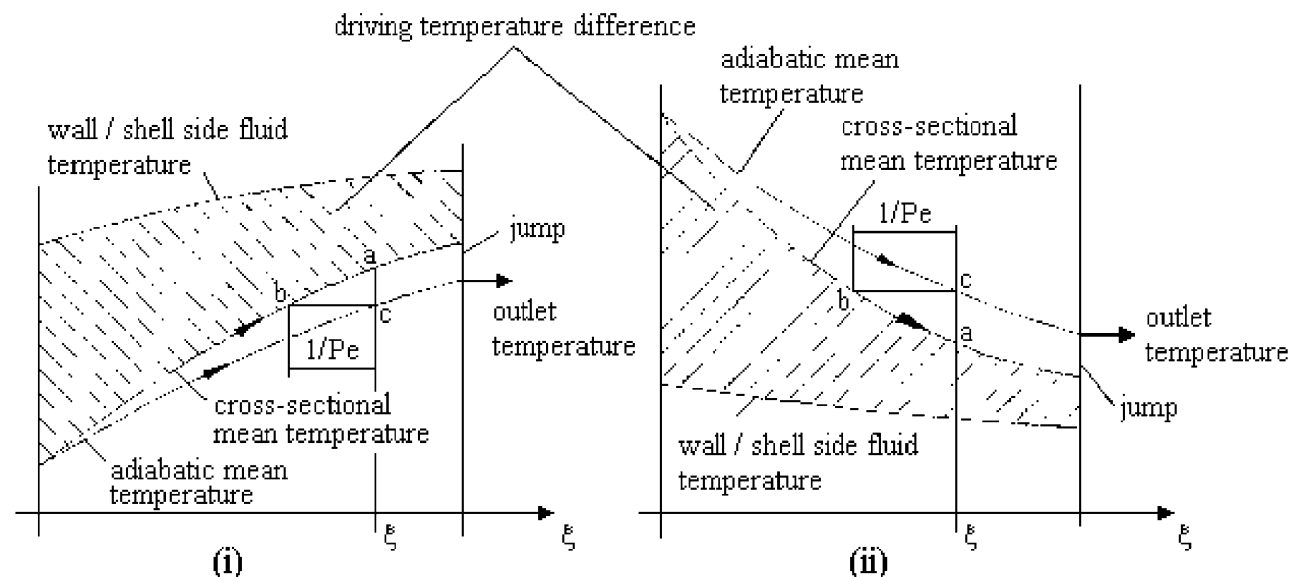

Fig. 5. Cross-sectional and adiabatic mean temperature profiles: (i) heated fluid and (ii) cooled fluid. 
Chester's flux equation can be developed by using Taylor's expansion of Eq. (29) and neglecting the second and higher order terms in flux to yield

$\varphi+\frac{M^{2}}{P e} \frac{\mathrm{d} \varphi}{\mathrm{d} \xi}=-\frac{\mathrm{d} \theta_{1 m}}{\mathrm{~d} \xi}$.

In the present context $M^{2}$ is a weighted parameter, but it has a broader meaning of "third sound wave Mach number" [17-19]. The existence of a hyperbolic model depends on the evaluation of $M^{2}$ from the constitutive equations of heat exchanger which is derived later. Similar to the parabolic model, the associated Peclet number in hyperbolic model, $P e_{\text {hyp }}$ can be determined from the energy balance.

\section{Determination of Mach number}

The evaluation of the weighted parameter, Mach number is essential in order to determine the model and its boundary conditions. The hyperbolic flux formulation given by Eq. (30) and flux expression Eq. (23) can be combined to yield

$$
\begin{aligned}
P e & \frac{1}{N} \sum_{j=1}^{N}\left(\frac{w_{1 j}}{\bar{w}_{1}}-1\right) \theta_{1 j}+M^{2} \frac{1}{N} \sum_{j=1}^{N}\left(\frac{w_{1 j}}{\bar{w}_{1}}-1\right) \frac{\mathrm{d} \theta_{1 j}}{\mathrm{~d} \xi} \\
= & -\frac{\mathrm{d} \theta_{1 m}}{\mathrm{~d} \xi} .
\end{aligned}
$$

Also substituting the second and third terms of Eq. (31) by Eq. (2) results in

$$
\begin{gathered}
P e \sum_{j=1}^{N}\left(\frac{w_{1 j}}{\bar{w}_{1}}-1\right) \theta_{1 j}+N T U_{1} M^{2} \sum_{j=1}^{N}\left(\frac{\bar{w}_{1}}{w_{1 j}}-1\right) \\
\left(\theta_{1 j}-\theta_{2}\right)=N T U_{1} \sum_{j=1}^{N} \frac{\bar{w}_{1}}{w_{1 j}}\left(\theta_{1 j}-\theta_{2}\right) .
\end{gathered}
$$

For no back flow at $\xi=0$, application of Eq. (16) yields that the first term of Eq. (32) is to be zero. Thus at $\xi=0$, Eq. (32) can be expressed as

$M^{2}=\frac{1}{N} \sum_{j=1}^{N} \frac{\bar{w}_{1}}{w_{1 j}} / \frac{1}{N} \sum_{j=1}^{N}\left(\frac{\bar{w}_{1}}{w_{1 j}}-1\right)$.

This equation has also been derived previously [19]. For the presence of back flow, Eq. (19) implies that the first term in Eq. (32) is also zero at $\xi=1$. Hence the expression for $M^{2}$ leads to same form as Eq. (33). Since the velocities in some of the tubes are negative, $M^{2}$ will have a fractional value. The expression for Mach number is an evidence for the existence of hyperbolic model. Eq. (33) indicates that though the expression is the same for both Mach numbers but its values are different depending on the flow situation in tube side. As per assumption that Mach number is a constant weighted parameter, its determination from the boundaries is also valid for the entire spatial coordinate.

The forward flow in the tubes gives $M^{2}>1$ and the partial backward flow gives $M^{2}<1$. Hence it is logical to assume that $M^{2}$ is unity when there is flow stagnation in the tube bundle without affecting the shell side flow. There are two such flow stagnation situations. The first case is one extreme condition when there is neither forward flow nor backward flow in the tube bundle and the second case is another extreme condition when the amount of fluid flow in forward direction returns back as the back flow. In both cases no fluid leaves the exit bonnet. The first one refers to $M \rightarrow 1^{+}$where the second one refers to $M \rightarrow 1^{-}$. These two values of Mach numbers are the limiting values of the two different flow situations. Mathematically, this leads to two different values having discontinuity between them. This discontinuity of Mach number at unity is quantitatively shown elsewhere [18].

Experimental evidence of Mach number. The adiabatic thermal environment can be created from an analogous mass transfer where the heat interaction between tube side and shell side fluids can be eliminated. This will lead both $N T U_{1}$ and $N T U_{2}$ to zero. By introducing a tracer at the inlet and from residence time measurement, Mach number can be calculated. For a pure maldistribution, $P e_{\text {hyp }}$ is also zero. Roetzel and $\mathrm{Na}$ Ranong [19] have calculated the Mach number from mass transfer experiment which exactly matches with the theoretical calculation given by Eq. (33). The experiment indicates that Mach number is not a fictitious quantity rather it is an independent quantity having experimental evidence.

\section{Derivation of boundary conditions}

For hyperbolic model, Eqs. (22) and (30) can be used to describe the cross-sectional mean temperature profile for tube side flow. Eq. (21) which is unchanged gives the actual (or cross-sectional mean) temperature profile of the shell side fluid. These three equations are the governing equations of the hyperbolic model of the heat exchanger. It has been observed that hyperbolic model is the only alternative due to the existence of finite, nonnegative value of Mach number. If hyperbolic model is the best representation of a cross-sectional mean temperature profile, the constitutive equations of heat exchanger and its associated boundary conditions must yield the hyperbolic type boundary and exit conditions. It may be noted that general hyperbolic boundary conditions have been proposed by Sahoo and Roetzel [18], where Danckwerts [3] boundary condition happens to be a special case as $M=0$.

Subsonic zone $(0 \leqslant M<1)$. In the subsonic zone, both forward and backward fluid flows are present in 
the tube side. By substituting Eq. (23) into Eq. (30) leads to

$P e_{\text {hyp }}\left(\theta_{1 f}-\theta_{1 m}\right)+\frac{M^{2}}{P e_{\text {hyp }}} \frac{\mathrm{d} \varphi}{\mathrm{d} \xi}=-\frac{\mathrm{d} \theta_{1 m}}{\mathrm{~d} \xi}$.

Substituting Eq. (22) into Eq. (34) results in

$$
\begin{aligned}
& \frac{M^{2}-1}{P e_{\text {hyp }}} \frac{\mathrm{d} \theta_{1 m}}{\mathrm{~d} \xi}+\frac{M^{2}}{P e_{\text {hyp }}} N T U_{1}\left(\theta_{1 m}-\theta_{2}\right) \\
& \quad=\left(\theta_{1 f}-\theta_{1 m}\right) .
\end{aligned}
$$

At the inlet $\xi=0$, the adiabatic mean temperature is the inlet temperature, $\theta_{1}^{-}$(equal to unity in the present case) of the tube side fluid. This fact can be easily verified from Eq. (18) and also from Fig. 3. Thus the inlet boundary condition from Eq. (35) can be written as

$$
\begin{aligned}
\xi=0: \quad & \frac{M^{2}-1}{P e_{\text {hyp }}} \frac{\mathrm{d} \theta_{1 m}}{\mathrm{~d} \xi}+\frac{M^{2}}{P e_{\text {hyp }}} N T U_{1}\left(\theta_{1 m}-\theta_{2}\right) \\
& =\left(\theta_{1}^{-}-\theta_{1 m}\right) .
\end{aligned}
$$

At the outlet $\xi=1$, the adiabatic and the cross-sectional mean temperatures are equal which can be seen from Eq. (19) and also from Fig. 3. Hence the exit boundary condition can be derived from Eq. (35) as

$\xi=1: \quad \frac{\mathrm{d} \theta_{1 m}}{\mathrm{~d} \xi}=-\frac{M^{2}}{M^{2}-1} N T U_{1}\left(\theta_{1 m}-\theta_{2}\right)$.

Supersonic zone $(M>1)$. In the supersonic zone, all the tube side fluid flows along the $\xi$ coordinate. As explained elsewhere [18], two boundary conditions are to be specified at the inlet.

The first inlet boundary condition at $\xi=1$ can be obtained from Eq. (16) (or Fig. 2) as

$\xi=0: \quad \theta_{1 m}=\theta_{1}^{-}=1$.

Eq. (16) (or Fig. 2) also indicates that cross-sectional and adiabatic mean temperatures are equal at $\xi=0$, if there is no back flow in the tube side. Hence from Eq. (35), the second inlet boundary conditions can be derived as

$\xi=0: \quad \frac{\mathrm{d} \theta_{1 m}}{\mathrm{~d} \xi}=-\frac{M^{2}}{M^{2}-1} N T U_{1}\left(\theta_{1 m}-\theta_{2}\right)$.

In the absence of back flow, Fig. 2 shows that the fluid exit temperature is $\theta_{1 f}$ which is different from $\theta_{1 m}$. Although this is not a boundary condition, but it is an important exit condition which can be derived from Eq. (35) as

$$
\begin{gathered}
\xi=1: \quad \frac{M^{2}-1}{P e_{\text {hyp }}} \frac{\mathrm{d} \theta_{1 m}}{\mathrm{~d} \xi}+\frac{M^{2}}{P e_{\text {hyp }}} N T U_{1}\left(\theta_{1 m}-\theta_{2}\right) \\
=\left(\theta_{1 f}-\theta_{1 m}\right) .
\end{gathered}
$$

Sonic zone $(M=1)$. The value of Mach number at unity refers to two stagnation situations as explained earlier.
In both the situations, the inlet fluid temperature to tube bundle is always the fluid temperature entering the bonnet. There is no exit boundary condition for fluid in tubes as in one case there is no flow and in other case the same fluid returns back. Thus for sonic flow there will be only one boundary condition [18] on cross-sectional mean temperature as

$\xi=0: \quad \theta_{1 m}=\theta_{1}^{-}=1$.

The exit condition on cross-sectional mean temperature which is not a boundary condition can be written as

$\xi=1: \quad \theta_{1 m}=\theta_{1}^{+}$.

The cross-sectional mean temperature at $\xi=0^{+}$will drop suddenly due to mixing at the inlet bonnet and at $\xi=1^{+}$, it will rise suddenly at the outlet bonnet due to a virtual mixing. For finite values of temperature as given by Eqs. (41) and (42), the drop or rise in mean temperature must be exponential in nature [18].

The boundary conditions and exit condition given by Eqs. (36)-(42) are resulted from the original governing equations of heat exchanger when the dispersive flux is formulated as a hyperbolic one. These equations are same as those derived by Sahoo and Roetzel [18] entirely from a different approach. On comparison, the lateral heat flux in [18] is equal to the lateral heat flux, $-N T U_{1}\left(\theta_{1 m}-\theta_{2}\right)$ of the present investigation. For parabolic model, Eqs. (36) and (37) reduce to Danckwerts boundary conditions [3] as $M=0$.

Estimation of dispersive Peclet number. The Peclet number $\left(P e_{\text {par }}\right.$ or $\left.P e_{\text {hyp }}\right)$ represents the dispersion values as it is not based on the diffusion of heat. These parameters are associated with dispersion flux which is a correction term in Eq. (22). Hence they must be determined from energy balance involving both the tube side and shell side fluids. The solution of cross-sectional mean temperatures of tube side and shell side fluids at the boundaries are enabled to estimate Peclet numbers. For subsonic zone, Eq. (36) can be used to determine $P e_{\text {hyp }}$, since Eq. (37) is independent of it. Similarly, for supersonic zone, Eq. (40) can be used to evaluate $P e_{\text {hyp }}$, since Eqs. (38) and (39) are independent of this parameter. For parabolic model $(M=0)$, only Eq. (36) can give the estimate of $P e_{\text {per }}$.

\section{Comparison of models with numerical calculation}

The constitutive equations for hyperbolic model are described by Eqs. (21), (22) and (30) with their boundary conditions, Eqs. (36)-(39). The solution of these equations should exactly depict the actual calculation of cross-sectional mean temperature profile in a heat exchanger. The numerical calculations done by Roetzel and Na Ranong [19] for actual heat exchanger, hyperbolic and parabolic models are shown in Figs. 6 and 7. 

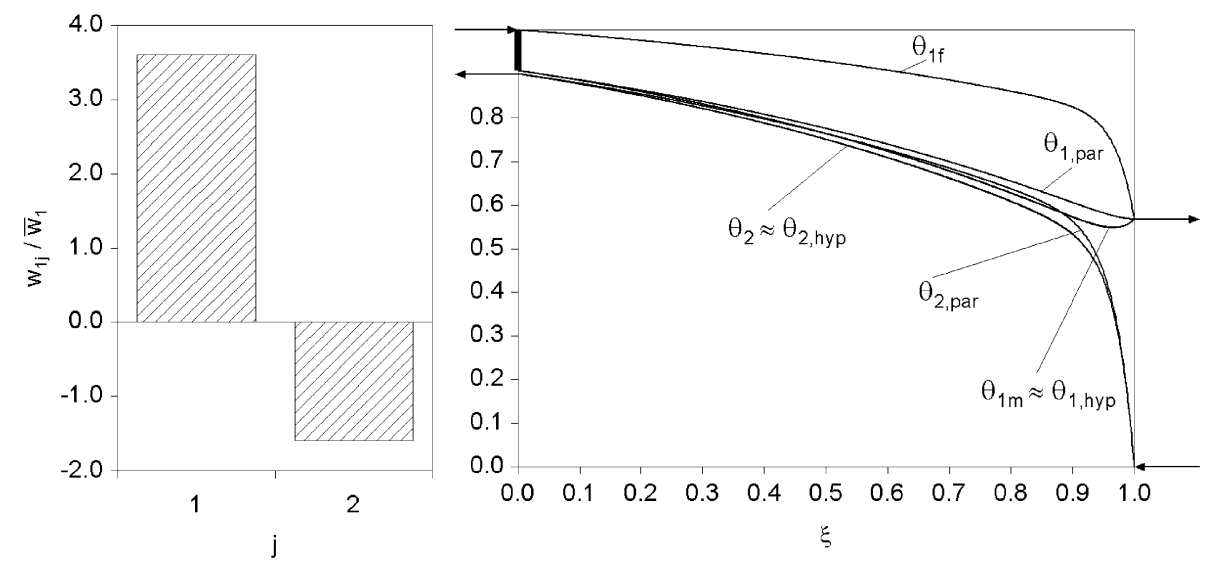

Fig. 6. Subsonic temperature profiles in axial dispersion models for two channels with back flow in one channel, $N T U_{1}=14.4$ and $N T U_{2}=30$.
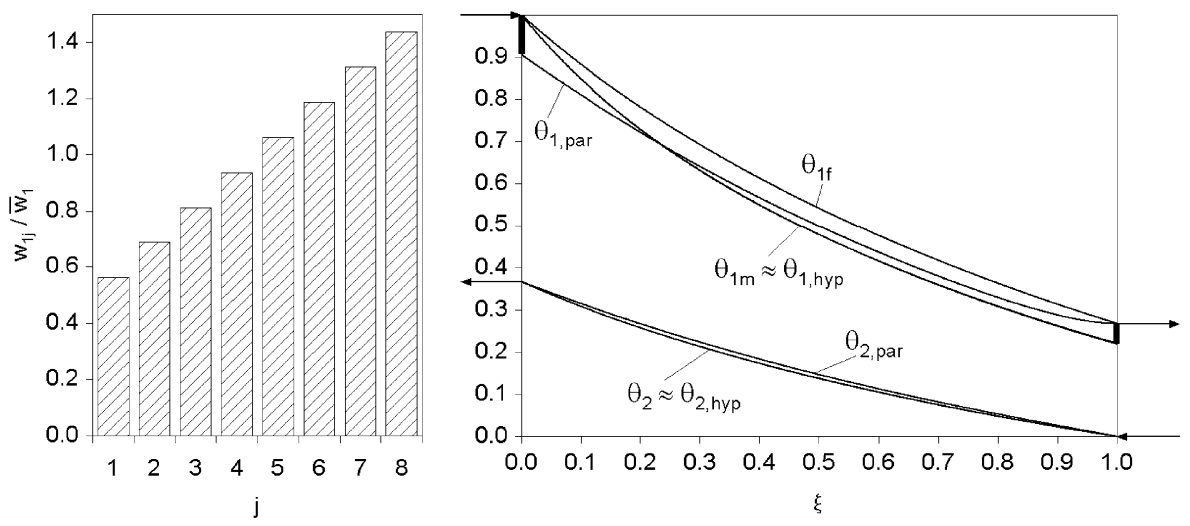

Fig. 7. Supersonic temperature profiles in axial dispersion models for quadratic velocity distribution, $N T U_{1}=2$ and $N T U_{2}=1$.

In their pioneering studies the boundary conditions for hyperbolic and parabolic models are prescribed in terms of flux. As shown in Fig. 6, the temperature jumps at the inlet and positive slope at the exit are the characteristic nature of subsonic boundary conditions described by Eqs. (36) and (37). In Fig. 7, the smooth nature of inlet temperature profile is due to the inlet boundary conditions described by Eqs. (38) and (39), and also the temperature jump at the exit is due to exit condition given by Eq. (40).

\section{Summary and conclusion}

An exhaustive theoretical foundation has been laid in this paper for recent developments in studies regarding wave model of axial dispersion. The maldistribution in the form of velocity non-uniformity can be accurately described if the dispersion flux behaves like Chester-type equation in exchange with Fourier's law.
The fundamental equations of hyperbolic model and its boundary conditions in terms of cross-sectional mean temperature have been derived from the basic equations of heat exchanger. This paper brings out the following factors:

- The cross-sectional mean temperature with maldistribution in a heat exchanger can be accurately predicted with a hyperbolic model.

- The Mach number in a hyperbolic model can predict back flow and flow stagnation.

- The Mach number for propagation of third sound wave can be estimated both theoretically and experimentally.

- The boundary conditions derived in this paper are exactly same as those proposed by Sahoo and Roetzel [18].

The above salient features establish the "third sound wave propagation" [17-19]. However, more investigations are required for the transient processes and the experimental determination of model parameters. 


\section{Acknowledgements}

This paper has been written during a research stay of the first author as visiting professor at the Institute of Thermodynamics of the University of Federal Armed Forces Hamburg. The financial support given to the first author by the University and the German Academic Exchange Service (DAAD) is gratefully acknowledged.

\section{References}

[1] S.G. Taylor, Dispersion of soluble matter in solvent flowing slowly through a tube, Proc. Royal Soc. London A 219 (1953) 186-203.

[2] S.G. Taylor, The dispersion of matter in turbulent flow through a pipe, Proc. Royal Soc. London A 223 (1954) 446-468.

[3] P.V. Danckwerts, Continuous flow systems - distribution of residence times, Chem. Eng. Sci. 2 (1953) 1-13.

[4] C.W. Rodehorst, V.J. Law, A one dimensional dispersion model for transient heat transfer in turbulent pipe flow, in: Proceedings of the 5th International Heat Transfer Conference, Paris, 1970.

[5] L.V. Beckman, V.T. Law, R.V. Bailey, D.U. van Rosenberg, Axial dispersion for turbulent flow with a large radial heat flux, AIChE J. 36 (1963) 598-604.

[6] J.C. Mecklenburgh, S. Hartland, The Theory of Backmixing, Wiley, London, 1975.

[7] M. Chester, Second sound in solids, The Phys. Rev. 131 (1963) 2013-2015.

[8] W. Roetzel, B. Spang, Axiales Dispersionsmodell für Regeneratoren mit endlicher Ausbreitungsgeschwindigkeit thermischer Störungen, Mitteilung aus dem Institut für Thermodynamik, Nr. 1, Universität der Bundeswehr Hamburg, 1994.
[9] W. Roetzel, B. Spang, Axiales Dispersionsmodell für Wärmeübertrager mit endlicher Ausbreitungsgesch windigkeit thermischer Störungen, Uniforschung, Forschungsmagazin der Universität der Bundeswehr Hamburg, 1993/ 1994, pp. 37-47.

[10] M.N. Ozisik, D.Y. Tzou, On the wave theory in heat conduction, J. Heat Transfer 116 (1994) 526-535.

[11] R.K. Sahoo, Propagation of thermal waves with lateral heat transfer, Cryogenics 34 (1994) 203-212.

[12] K.R. Westerterp, V.V. Dil'man, A.E. Kronberg, Wave model for longitudinal dispersion: development of the model, AIChE J. 41 (1995) 2013-2028.

[13] K.R. Westerterp, V.V. Dil'man, A.E. Kronberg, A.H. Benneker, Wave model for longitudinal dispersion: analysis and applications, AIChE J. 41 (1995) 2029-2039.

[14] A.E. Kronberg, A.H. Benneker, K.R. Westerterp, Wave model for longitudinal dispersion: application to the laminar-flow tubular reactor, AIChE J. 42 (1996) 31333145.

[15] A.H. Benneker, A.E. Kronberg, K.R. Westerterp, Longitudinal mass and heat dispersion in tubular reactors, Indust. Eng. Chem. Res. 36 (1997) 2031-2040.

[16] W. Roetzel, S.K. Das, Hyperbolic axial dispersion model concept and its application to a plate heat exchanger, Int. J. Heat Mass Transfer 38 (1995) 3065-3076.

[17] W. Roetzel, B. Spang, X. Luo, S.K. Das, Propagation of the third sound wave in fluid: hypothesis and theoretical foundation, Int. J. Heat Mass Transfer 41 (1998) 27692780.

[18] R.K. Sahoo, W. Roetzel, Boundary conditions for hyperbolic dispersion: an analytical foundation of the propagation of third sound wave, Research Report, Institut für Thermodynamik, Universität der Bundeswehr Hamburg, October 2000.

[19] W. Roetzel, Ch. Na Ranong, Axial dispersion models for heat exchangers, Int. J. Heat Technol. 18 (2000) 7-17. 